\title{
Accumulation of Mononucleotides in Washed Suspensions of Myxamoebae of Dictyostelium discoideum
}

\author{
By M. I. KRICHEVSKY AND L. L. LOVE \\ U.S. Department of Health, Education, and Welfare, Public Health Service, \\ National Institutes of Health, National Institute of Dental Research, \\ Bethesda, Maryland 20014, U.S.A.
}

(Accepted for publication 27 June 1967)

SUMMARY

Washed myxamoebae of Dictyostelium discoideum exhibit a transitory accumulation of $2^{\prime}, 3^{\prime}$-mononucleotides in the soluble pool. The accumulation is correlated with the time of transition from the vegetative stage to the initiation of morphogenesis. Materials which stimulate the rate of morphogenesis and prevent the efflux of RNA and protein from washed amoebae, also enhance the accumulation of mononucleotides many fold.

\section{INTRODUCTION}

Previous communications have described the ability of a variety of materials to stimulate the rate of morphogenesis in the cellular slime mould Dictyostelium discoideum (Krichevsky \& Wright, 1963). Further investigation of one class of stimulatory materials (i.e. imidazole-containing compounds) led to the postulate that the site of action of these materials is at the surface of the organisms (Krichevsky \& Love, 1964). Stimulants of the rate of development prevented the specific efflux of RNA and protein into the extracellular environment of washed myxamoebae (Krichevsky \& Love, 1965). It is well documented (Wright, 1964) that endogenous RNA and protein concentrations decrease in developing cells. Evidence is presented here that the monomeric constituents of one of these macromolecules, RNA, accumulated in washed suspensions of $D$. discoideum myxamoebae under the influence of various stimulants of the rate of morphogenesis.

\section{METHODS}

The procedures for growing and harvesting the myxamoebae by using a solid medium were as described previously (Krichevsky \& Wright, 1963). The method of Gerisch (1959) was used for growing the myxamoebae in liquid culture. Whether grown on solid or in liquid media, the myxamoebae were washed, suspended and incubated as described by Krichevsky \& Love (1965).

Pentose-containing materials were assayed by the orcinol reaction of Mejbaum as modified by Horecker, Smyrniotis \& Klenow (1953). The ninhydrin assay for amino acids was performed by the procedure of Rosen (1957). Diphenylamine-reacting materials were determined as described by Dische (1955). 


\section{RESULTS}

As reported previously, L-histidine stimulated the rate of morphogenesis and prevented the efflux of macromolecules more effectively at higher hydrogen ion concentrations (e.g. $\mathrm{pH}$ 5) than at lower $\mathrm{H}^{+}$concentrations $\left(\mathrm{pH}_{7}\right)$. Therefore, it became of interest to ascertain the ability of L-histidine, at various $\mathrm{H}^{+}$concentrations, to promote the accumulation of perchloric acid-soluble pentose-containing materials $\left(2^{\prime}, 3^{\prime}-\right.$ mononucleotides, see below). The results are presented in Fig. I. When the suspending medium was L-histidine at $\mathrm{pH} 5$ and 6 the accumulation was markedly greater than when the medium was either L-histidine at $\mathrm{pH} 7$ or distilled water. Since urocanic acid

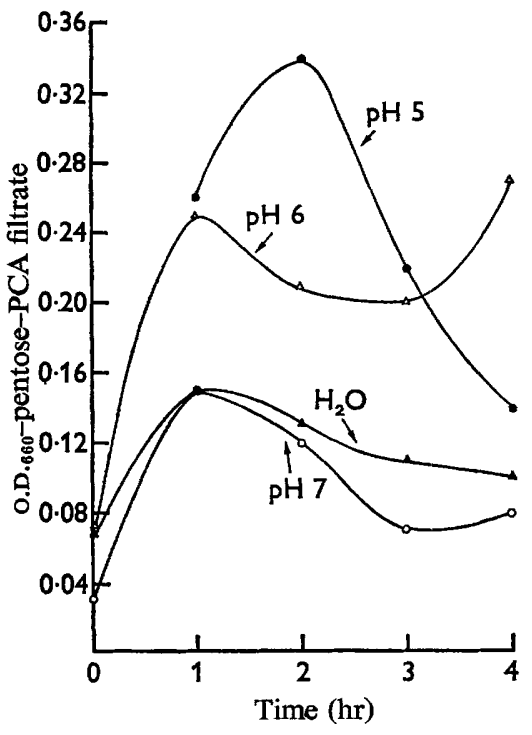

Fig. I

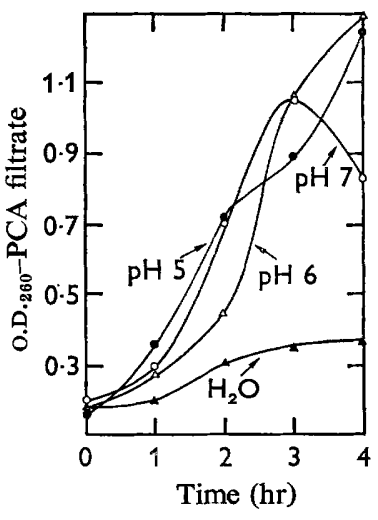

Fig. 2

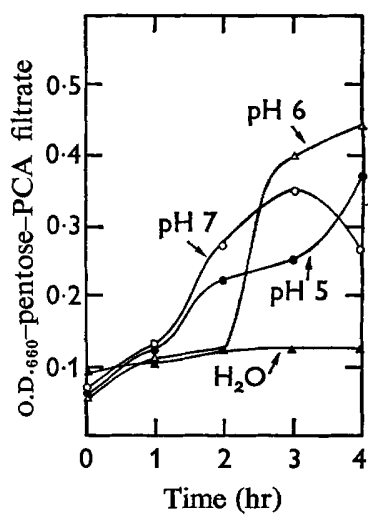

Fig. I. Orcinol-reactive material accumulation in the presence of histidine at various hydrogen ion concentrations. Myxamoebae of Dictyostelium discoideum, harvested after growth on solid medium, were suspended in $0.04 \mathrm{M}-\mathrm{L}-$ histidine $(\mathrm{pH}, 0-\mathrm{O} ; \mathrm{pH} 6, \triangle-\triangle$; $\mathrm{pH} 7, \mathrm{O}-\mathrm{O})$ or distilled water $(\boldsymbol{\Delta}-\mathbf{\Delta})$. At the times indicated, $2.0 \mathrm{ml}$. samples were added to $0.2 \mathrm{ml}$. $N$-perchloric acid (PCA), chilled in an ice bath, and filtered through $25 \mathrm{~mm}$. diam. Millipore filters (type HA, $0.45 \mu$ pore size). One ml. samples of the filtrate were assayed for their pentose-containing materials.

Fig. 2. Accumulation of orcinol-reactive and ultraviolet absorbing materials in the presence of potassium phosphate buffers. The conditions of incubation of the Dictyostelium discoideum myxamoebae were the same as described for Fig. I. The suspending media were I·OM potassium phosphate buffers $(\mathrm{pH} 5,-0 ; \mathrm{pH} 6, \triangle-\triangle ; \mathrm{pH}, 0-\mathrm{O})$ or distilled water $(\Delta-\Delta)$. Before the perchloric acid (PCA) filtrates were assayed for pentose, the extinction was read at $260 \mathrm{~m} \mu$ (I cm. light path).

is a product of L-histidine metabolism in Dictyostelium discoideum (Krichevsky \& Love, I964) and this material has an absorption maximum at $260 \mathrm{~m} \mu$ in acid, the ultraviolet (u.v.) absorption changes in the samples reflected the urocanate production (ascertained by u.v. absorption at $300 \mathrm{~m} \mu$ in $0 . \mathrm{I} \mathrm{N}-\mathrm{NaOH}$; these are not presented).

The substitution of potassium phosphate buffers for the L-histidine solutions gave the data shown in Fig. 2. Qualitatively similar results were obtained whether the assay 
used was u.v. absorption (at $260 \mathrm{~m} \mu$ ) or reactivity with orcinol $+\mathrm{FeCl}_{3}$ reagent (for pentose moeities). In addition, potassium phosphate stimulated the accumulation of the assayed materials irrespective of the $\mathrm{pH}$ value; this correlated well with the ability of this material to stimulate morphogenesis at the various $\mathrm{pH}$ values.

Other materials which stimulate the rate of morphogenesis and inhibit leakage of macromolecules were tested for their ability to promote increased mononucleotide concentrations (Fig. 3). Of the compounds tested, glucose was by far the most effective; the values obtained having been divided by 3 to bring them within the scale shown. Magnesium ion was somewhat more stimulatory than $\mathrm{Na}^{+}$or $\mathrm{K}^{+}$.
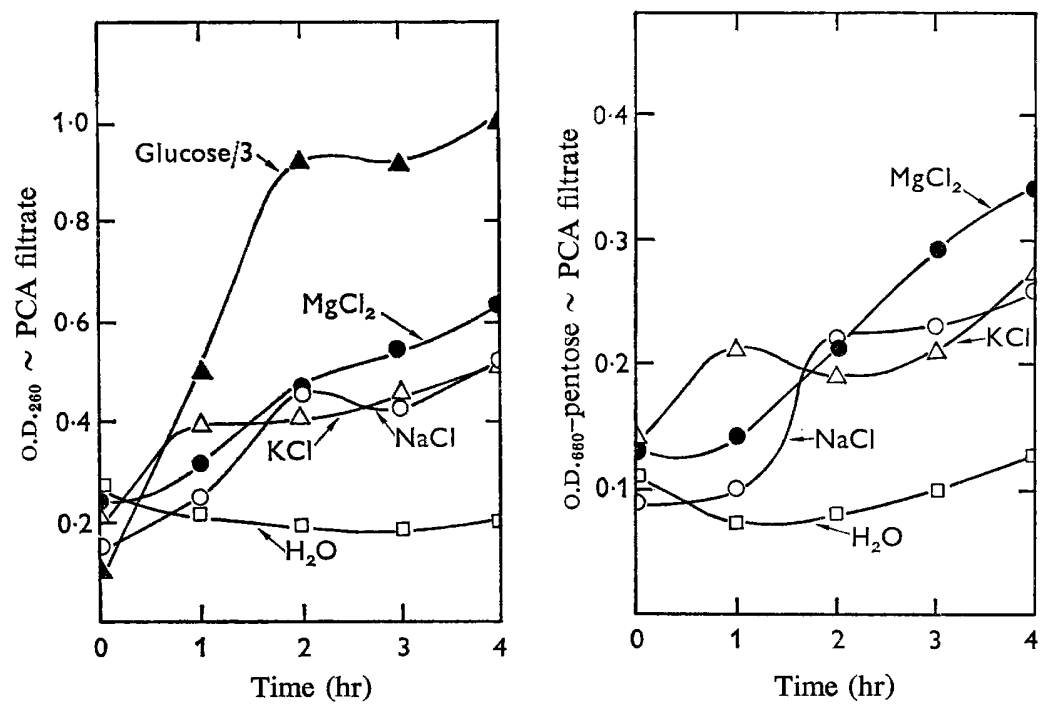

Fig. 3. Accumulation of orcinol-reactive and ultraviolet absorbing materials in the presence of chloride salts or glucose. The conditions for incubation of the Dictyostelium discoideum myxamoebae were the same as those described for Fig. I. The myxamoebae were suspended in the following media: $0.0 \mathrm{IM}-\mathrm{NaCl}(\mathrm{O}-\mathrm{O}) ; 0.01 \mathrm{M}-\mathrm{KCl}(\triangle-\triangle) ; 0.005 \mathrm{M}-\mathrm{MgCl}_{2}(\mathbf{O}-\mathrm{O})$ $0.05 \mathrm{M}$-glucose $(\Delta-\Delta)$; and $\mathrm{H}_{2} \mathrm{O}(\square-\square)$. Before the perchloric acid (PCA) filtrates were assayed for pentose, the extinction was read at $260 \mathrm{~m} \mu$ ( $\mathrm{I} \mathrm{cm}$. light path).

Experiments similar to those described above indicated that imidazole in the suspending medium also resulted in increased mononucleotide concentrations. To relate the accumulation phenomenon to the morphogenetic process, the experiment described in Fig. 4 was performed. By both assay procedures it was shown that the stage of development had a marked influence on the results. At the earlier stage, accumulation was observed when either distilled water or imidazole solution was the suspending medium. However, imidazole was more effective in promoting accumulation, and with it maximal accumulation occurred at the early aggregative stage.

Figure 5 shows the effect of developmental stage on the kinetics of amino nitrogen accumulation under the conditions of the experiment just described. Even over the full $4 \mathrm{hr}$ of incubation no particular influence of either morphogenetic stage or suspending medium was evident when compared to the results obtained when mononucleotides were assayed as described for Fig. 4.

Myxamoebae which had been grown in liquid culture in which the only nutrients 
were Escherichia coli organisms and phosphate buffer exhibited a marked change in accumulating ability with changing developmental stage (Fig. 6). Thus, only the vegetative myxamoebae (the freshly harvested forms) were able to accumulate mononucleotides. As shown by extinction measurements at $260 \mathrm{~m} \mu$, the accumulation by the myxamoebae was dependent on the presence of phosphate. The accumulation of orcinol-reactive material in the absence of phosphate (i.e. in the water control sample) was found only in this experiment. The material presumably was not purine or
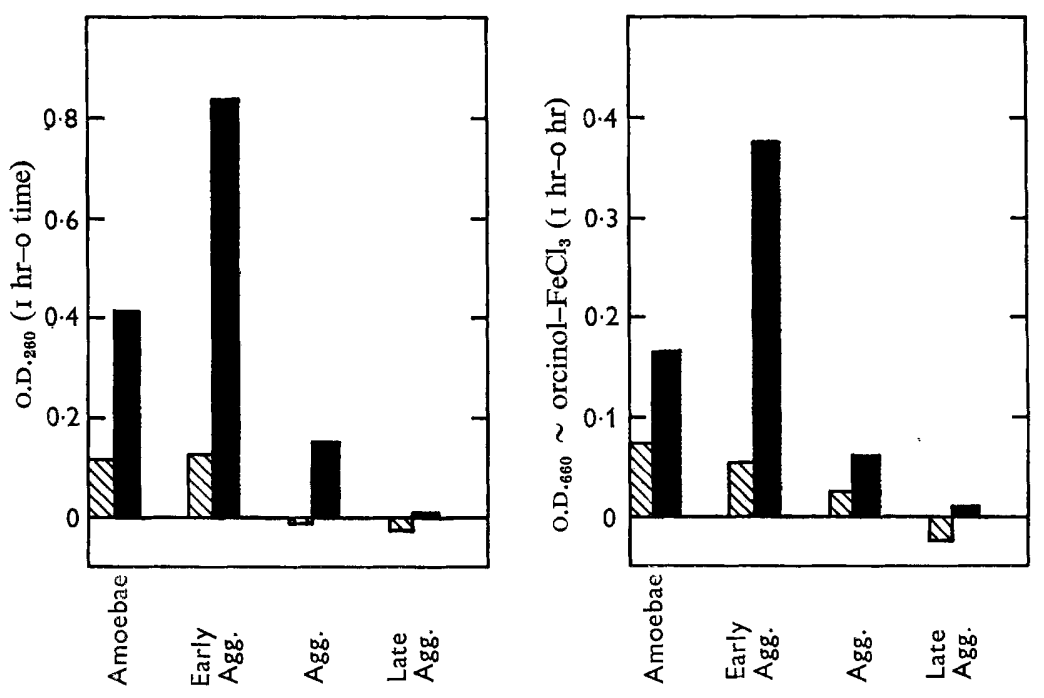

Fig. 4. Effect of developmental stage on the accumulation with imidazole as the stimulant. Myxamoebae of Dictyostelium discoideum were grown on solid medium contained in cookie sheets. At intervals through the growth period, groups of cookie sheets were transferred from incubation at $22^{\circ}$ to incubation at $19^{\circ}$. This procedure made possible the selection of groups of sheets which, on visual inspection, were determined to be at the developmental stages of vegetative amoebae (amoebae), early aggregation (early agg.), mid-aggregation (agg.), and late aggregation (late agg.). Four suspensions were prepared in the usual manner; one from organisms at each developmental stage. The cell concentrations were adjusted to the same extinctions (I/15 dilution $=0.55$ at $660 \mathrm{~m} \mu ; \mathrm{I} \mathrm{cm}$. light path). The organisms were then further diluted $(\mathrm{I} / \mathrm{IO})$ in either $0.05 \mathrm{M}$-imidazole, $\mathrm{pH} 6(\mathbb{C})$, or distilled water $(\mathbb{\nabla})$. The conditions of incubation and assays were the same as described for Fig. 2. However, the data presented here are the changes of the extinction at $260 \mathrm{~m} \mu$, or the pentose-moiety contents of the perchlorate-soluble fractions for the first hour only.

pyrimidine in nature since there was no corresponding increase in u.v. absorption. When glucose was used instead of phosphate, the results were like those just described (Fig. 7), indicating that phosphate was not unique in function.

Preliminary determinations of the nature and distribution of the u.v. absorbing orcinol-reactive materials confirmed the observation of Pannbacker (1966) for normal pools as follows. The great majority of the $260 \mathrm{~m} \mu$ absorption was due to materials which behaved like the $2^{\prime}$ - or $3^{\prime}$-mononucleotide constituents of RNA when eluted from Sephadex A-25 ion exchange columns. The isolated materials had u.v. absorption spectra which were identical with adenosine, cytosine, uridine and guanosine-2', $3^{\prime}-$ monophosphates. Thin-layer or paper chromatography on polyethylene-iminecellulose indicated that the isolated materials behaved like the $2^{\prime}$ - or $3^{\prime}$-mononucleotides 
rather than the 5 -esters. In addition, they were resistant to periodate oxidation under conditions which cleaved the ribose moiety of the 5 -esters. Quantitative assays for diphenylamine reactivity showed that the samples did not contain significant amounts of deoxyribotides.
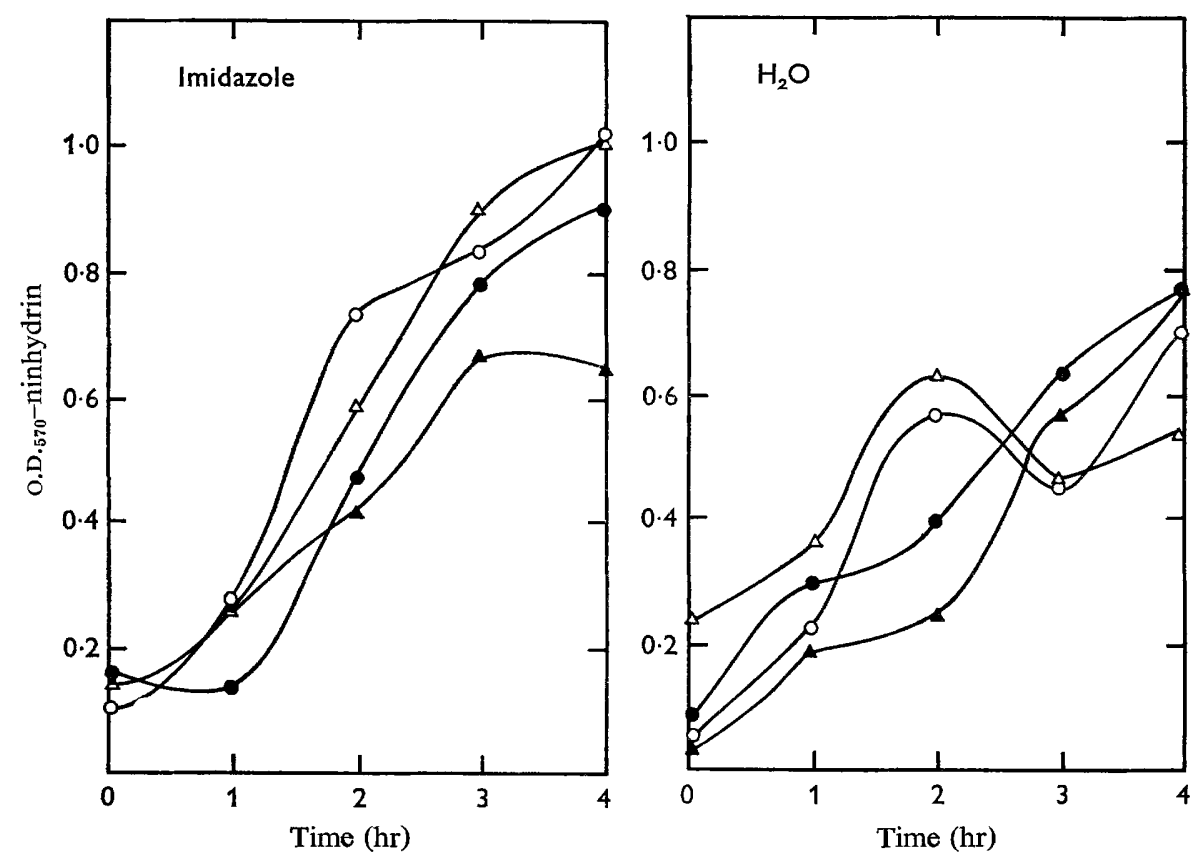

Fig. 5. Amino-nitrogen accumulation, with developmental stage of Dictyostelium discoideum, in the presence and absence of imidazole. All conditions were those described for Fig. 4. The samples taken for the ninhydrin assay were $0.05 \mathrm{ml}$. In this case the data for the full $4 \mathrm{hr}$. incubation period is presented; imidazole on the left and $\mathrm{H}_{2} \mathrm{O}$ on the right. The developmental stages are: vegatative amoebae $(-0)$, early aggregation $(\mathrm{O}-\mathrm{O})$, mid-aggregation $(\triangle-\triangle)$, and late aggregation $(\boldsymbol{\Delta}-\mathbf{A})$.

\section{DISCUSSION}

In those cases tested, materials which have been found to stimulate the rate of sorocarp formation were shown to prevent the efflux of macromolecules from washed Dictyostelium discoideum myxamoebae (Krichevsky \& Love, 1965). The macromolecules specifically concerned were ribosenucleic acids and proteins. Thus, it was postulated 'that the RNA and protein that leak from the cells are not essential for cellular differentiation but provide stimulatory intermediates through their catabolism to their monomeric constituents, and this only when available inside the cell'. It might be expected that conditions leading to the internal conservation of RNA and protein might lead to an increased pool level of the monomers of these macromolecules. However, this would be true only if the rates of entry of the monomers into the pool exceed the rates of exit from the pool (either through further catabolism or leakage of the monomers themselves or both).

In the case of protein depolymerization, it appears that pool levels of amino acids are not affected markedly by materials which both stimulate the rate of morphogenesis 
and prevent the efflux of protein from amoebae under the conditions described. Further, it may be concluded that the pool levels of amino acids are unaffected by the stage of development (at least through aggregation). Thus, the rate of development probably is not controlled by pool levels of amino acids.

The picture is quite different when RNA monomers are considered. At the time when the amoebae are undergoing the transition from the growing stage to the developmental stage of the life cycle, there is a transitory accumulation of mononucleotides in the soluble pool. This accumulation is enhanced many fold by the presence of materials which stimulate the rate of morphogenesis. In addition, these
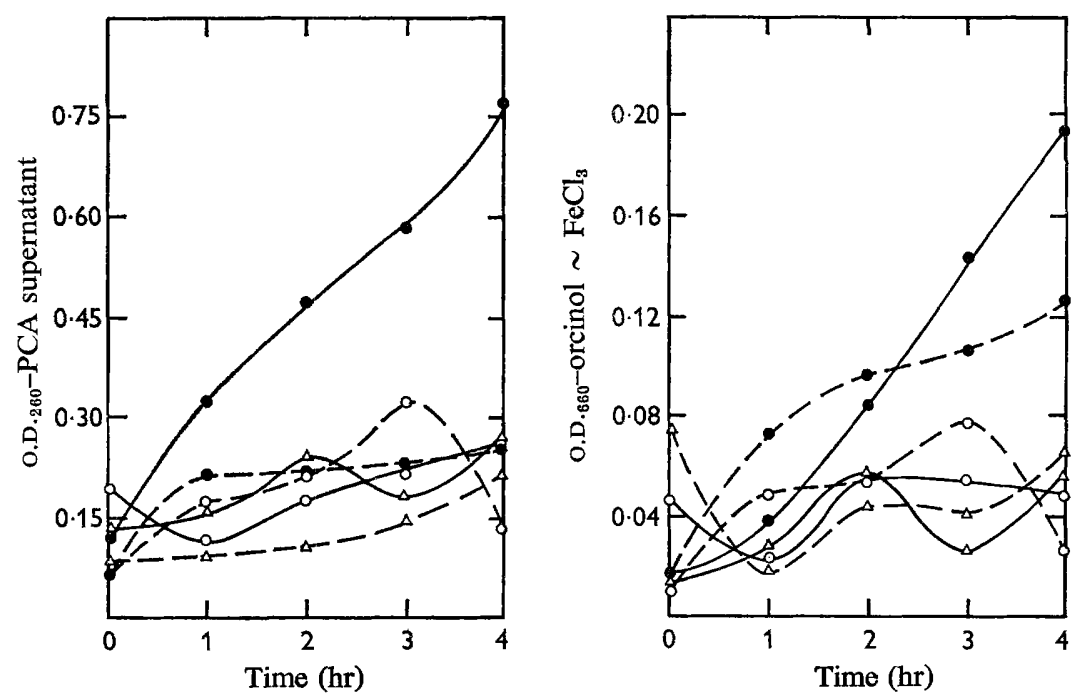

Fig. 6. Accumulation, with developmental stage, of orcinol-reactive and ultraviolet-absorbing materials by Dictyostelium discoideum grown in liquid culture. Myxamoebae were grown as described by Gerisch (1959); washed free of residual bacteria and six samples of the crop treated as follows. The first amoeba sample was suspended in a final concentration of O.IM-potassium phosphate buffer ( $\mathrm{pH}_{5}$ ) (-O); the second amoeba sample was suspended in distilled water ( - - - ) ; the remaining four samples were spread on cookie sheets containing $2 \%$ Difco-Noble agar and allowed to incubate until the desired developmental stage was judged to have been reached by visual inspection. The samples were then washed off the agar surface with distilled water and suspended for incubation, i.e. early aggregation: O.IM-phosphate $(\triangle-\triangle)$ or distilled water $(\triangle-. \triangle)$; mid-aggregation: 0.1 M-phosphate $(\mathrm{O}-\mathrm{O})$ or distilled water $\left(\mathrm{O}-\mathrm{O}^{-}\right.$). The suspensions were sampled and assayed as described for Fig. 2.

same materials allow the conservation of RNA within the cells. It may be postulated that the rate-limiting step (or steps) in the development of Dictyostelium discoideum will be found associated with the further utilization of one or more mononucleotides which, in turn, arise from the deploymerization of endogenous RNA. Further, it would be expected that exogenously supplied mononucleotides would stimulate the rate of morphogenesis. Preliminary experiments indicate that this is true, and at molar concentrations of as little as $10^{-4} \mathbf{M}$ which is at least 100 times as effective as the other stimulants found. However, the form of mononucleotides which is most effective is the $5^{\prime}$-ester. 


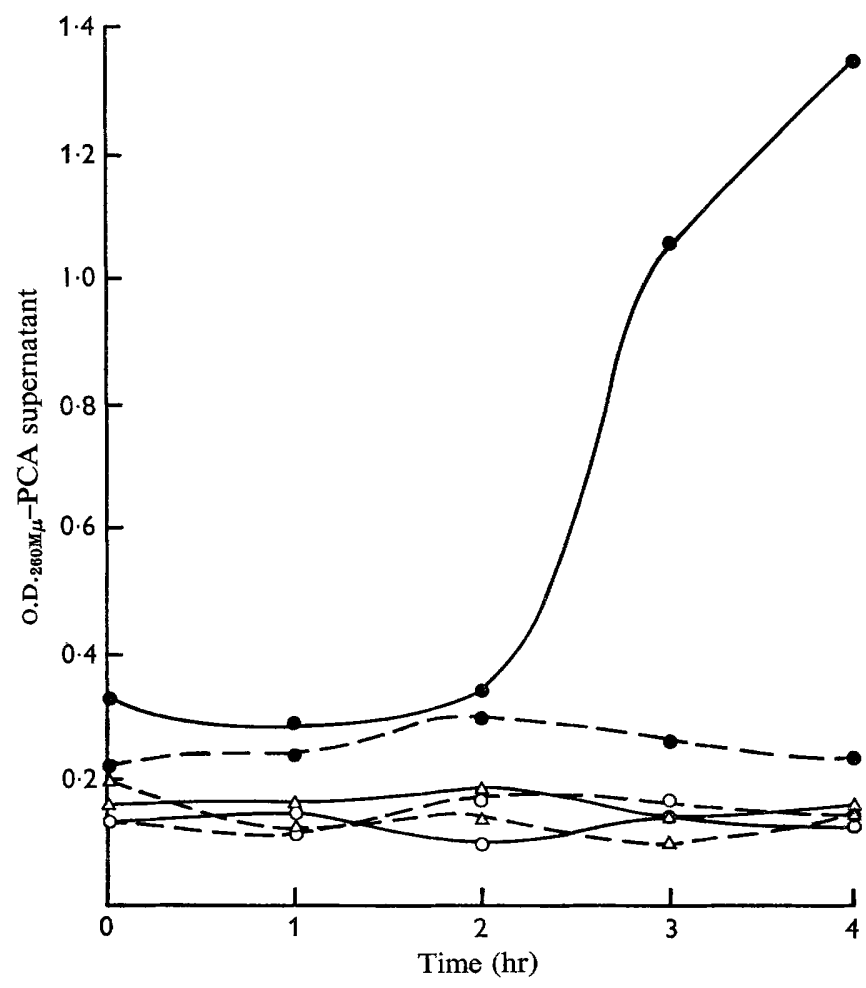

Fig. 7. Accumulation, with developmental stage, of ultraviolet absorbing materials by myxamoebae of Dictyostelium discoideum grown in liquid culture. All conditions were identical to those described in Fig. 6 except that $0.05 \mathrm{M}$-glucose was substituted for the O.IM-phosphate.

\section{REFERENCES}

Dische, Z. (1955). In The Nucleic Acids, vol. I. Ed. by E. Chagaff and J. N. Davidson. New York: Academic Press.

Gerisch, G. (1959). Ein Submerskultuver-fahren für entwicklungsphysiologische untersuchungen an Dictyostelium discoideum. Naturwissenschaften 46, 654 .

Horecker, B. L., SMYrniotis, P. Z. \& Klenow, H. (1953). The formation of sedoheptulose phosphate from pentose phosphate. J. biol. Chem. 205, 661 .

KRICHEVSKY, M. I. \& LOVE, L. L. (I964). The uptake and utilization of histidine by washed amoebae in the course of development in Dictyostelium discoideum. J. gen. Microbiol. 34, 483 .

KrichevsKy, M. I. \& Love, L. L. (1965). Efflux of macromolecules from washed Dictyostelium discoideum. J. gen. Microbiol. 47, 367.

KRICHEVSKY, M. I. \& WRIGHT, B. E. (1963). Environmental control of the course of development in Dictyostelium discoideum. J. gen. Microbiol. 32, 195.

PANnBaCker, R. G. (1966). RNA metabolism during differentiation in the cellular slime mold. Biochem. biophys. Res. Comm. 24, 340.

Rosen, H. (1957). A modified ninhydrin colormetric analysis for amino acids. Archs. Biochem. Biophys. 67, 10.

Wright, B. E. (1964). In Biochemistry and Physiology of Protozoa, Ed. by S. H. Hutner, vol. 3. New York: Academic Press 